
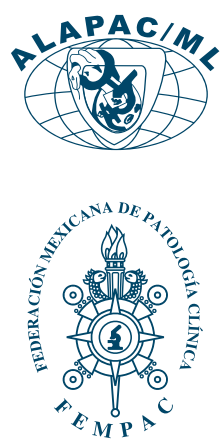

\title{
Protocolo del laboratorio SMNYL para la verificación de métodos inmunológicos cualitativos
}

\author{
SMNYL laboratory protocol for the verification \\ of qualitative immune methods
}

\author{
Alejandrez-Martínez N,* Hoyos-Torres L,* Saldaña-Sandria L ${ }^{\ddagger}$
}

Palabras clave: Acreditación, verificación, métodos cualitativos, concordancia.

Keywords: Accreditation, verification, qualitative test, agreement.

* Analista químico.

‡ Coordinadora del

Laboratorio Clínico.

Seguros Monterrey, New York Life

Correspondencia: Alejandrez-Martínez N Laboratorio Clínico, Departamento de Administración de Riesgos,

Seguros Monterrey New York Life, Tel. 52 4422384000, ext. 4368,

E-mail: nalejandrezm @mnyl.com.mx

Recibido: 02/03/2020 Aceptado: 02/04/2020

\section{RESUMEN}

La verificación de los métodos es un requisito indispensable para acreditar un ensayo en un sistema de gestión de calidad bajo la NMX-EC-15189-IMNC-2015; sin embargo, en México no existe un protocolo establecido o difundido por la entidad mexicana de acreditación para la verificación de métodos cualitativos, tal como lo establece para métodos cuantitativos, y las guías internacionales resultan inaccesibles para la mayoría de los laboratorios debido a los altos costos que representa su ejecución. Es por ello que el Laboratorio SMNYL documentó y ejecutó un protocolo propio, acorde con el uso previsto, para verificar las características de desempeño documentado por el fabricante y lograr la acreditación de tres ensayos inmunológicos cualitativos, lo que nos permite proponerlo como una alternativa, ya que a la par de ser sencillo y accesible, permite la obtención de evidencia objetiva para la verificación del desempeño.

\section{ABSTRACT}

The verification of the methods is an indispensable requirement to a test in a quality management system under NMXEC-15189-IMNC-2015, however in Mexico there is no protocol established by the Mexican Accreditation Entity for the verification of qualitative methods, as established for quantitative methods, and international guidelines are inaccessible to most laboratories due to the high costs of their execution. That is why the SMNYL Laboratory documented and executed its own protocol, in accordance with the intended use, to verify the performance characteristics documented by the manufacturer to achieve the accreditation of three qualitative immunological tests, which allows us to propose it as an alternative, as well as being simple and accessible, it allows obtaining objective evidence for performance verification.

\section{Definiciones:}

Acreditación: procedimiento por el cual un organismo autorizado reconoce formalmente a una organización como competente para llevar a cabo tareas específicas.

Certificación: procedimiento por el cual un tercero da garantía escrita de que un producto o servicio es conforme a requisitos específicos.

Métodos cualitativos: métodos de análisis cuya respuesta es la presencia o ausencia de un analito, detectado en forma directa o indirecta en una muestra, considerando sus propiedades físicas, biológicas o químicas.

Validación: confirmación mediante aportación de evidencia objetiva que se han cumplido los requisitos para una utilización o aplicación específica prevista.

Verificación: confirmación mediante la aportación de evidencia objetiva, con la realización de ensayos/pruebas y demostraciones de que se han cumplido los requisitos de desempeño especificados.

\section{INTRODUCCIÓN}

E n los últimos años la calidad en el laboratorio de análisis clínicos ha tomado particular importancia; ésta fomentada por la necesidad de tener resultados oportunos, confiables y trazables, características que satisfacen las necesidades de las partes involucradas y, sobre todo, contribuyen a un diagnóstico y/o tratamiento efectivo para la salud del paciente. La siste- 
matización de las actividades del laboratorio de análisis clínicos y la mejora continua de los procesos operativos y técnicos a través de un Sistema de Gestión de la Calidad (SGC) es la vía certera para conseguir que los resultados emitidos cumplan con dichas características.

Existen diferentes directrices que se pueden utilizar para la implementación de un SGC, destacando la Norma ISO 15189:2012, en su versión para México la NMX-EC15189-IMNC-2015, ${ }^{1}$ por ser específica para laboratorios clínicos ya que, además de los requisitos generales del SGC, estipula requisitos técnicos de calidad y competencia para los procesos centrales del laboratorio. Adicionalmente, los laboratorios que implementan SGC basados en esta norma son acreditables por la Entidad Mexicana de Acreditación (ema), ${ }^{2}$ lo que les otorga un reconocimiento a su calidad con estándares internacionales.

Si bien es cierto que desde que la ema comenzó con los procesos de evaluación y acreditación para laboratorios clínicos en 2005, el número de acreditados ha ido en franco crecimiento (Figura 1). ${ }^{3}$ En la actualidad, de los aproximadamente 15 mil laboratorios registrados ante la Comisión Federal para la Protección contra Riesgos Sanitarios (COFEPRIS), ${ }^{4}$ sólo 128 de ellos se encuentran acreditados, ${ }^{5}$ lo que representa menos de $1 \%$, una cifra que refleja los bajos índices de acreditación en México y que no son muy diferentes a los del resto de América Latina. ${ }^{6}$

Aunque se conocen bien los beneficios de la acreditación, es un proceso que los laboratorios perciben como lento, costoso y de muy alta dificultad, en mayor medida por el cumplimiento de los requisitos técnicos, con énfasis en los asociados al proceso de validación y verificación de los métodos de examen. ${ }^{7,8}$

El punto 5.5.1. de la NMX-EC-15189-IMNC-2015 determina que la validación y verificación de los métodos

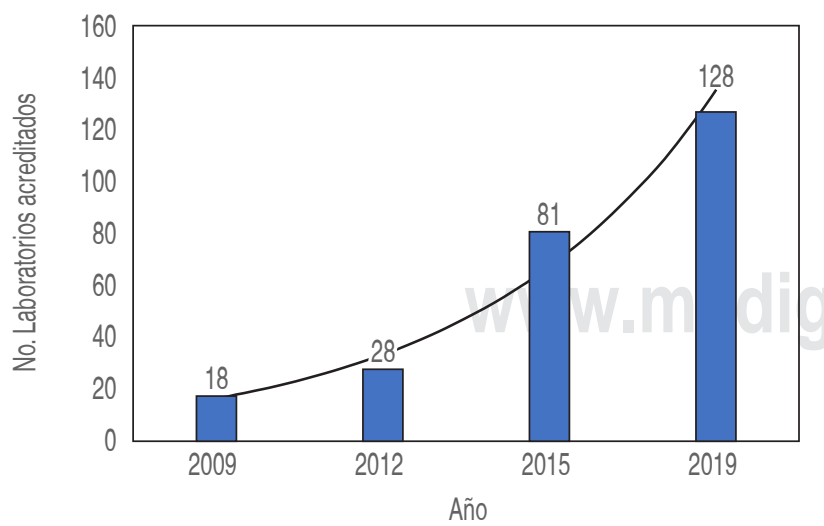

Figura 1: Crecimiento del número de laboratorios acreditados en México en los últimos 10 años. de examen debe ser realizada por el laboratorio con el objetivo de confirmar, mediante la obtención de evidencia objetiva, que los exámenes se desempeñan según las características establecidas por el laboratorio o el propio fabricante para el uso previsto.

La validación es necesaria cuando los métodos de ensayo son no normalizados, normalizados modificados o desarrollados por el propio laboratorio, y en ella se evalúan características como la selectividad/ especificidad, precisión, exactitud, linealidad, límite de detección, límite de cuantificación y robustez. ${ }^{9}$ La verificación es necesaria cuando el método de ensayo empleado por el laboratorio es normalizado y utilizado sin modificaciones, consiste en comprobar que el método se desempeña dentro de los parámetros documentados por el fabricante. En México, la ema en conjunto con el Centro Nacional de Metrología (CENAM) proporcionan una guía para la verificación de los procedimientos de examen cuantitativos, ${ }^{10}$ en la que se establece que se deben evaluar como mínimo las características de linealidad, precisión, veracidad e incertidumbre; sin embargo, para métodos cualitativos no existe una guía establecida por la ema, lo que deja a los laboratorios con el dilema de cómo verificar el desempeño de estos ensayos, y aunque existen guías internacionales como las del CLSI ${ }^{11}$ o EURACHEM ${ }^{12}$ resultan inaccesibles debido a los altos costos que representa en materiales, número de muestras e insumos necesarios. Es por ello que en el presente trabajo se presenta el protocolo que utilizó el Laboratorio de Seguros Monterrey New York Life (SMNYL) para lograr la acreditación de tres ensayos inmunológicos cualitativos, tomando como base los lineamientos establecidos por la asesora en calidad Alba Cecilia Garzón, ${ }^{13}$ lo cual robustece el análisis con la construcción de tablas de contingencia y el cálculo de concordancias que recomienda la Guía EP12-A2, ${ }^{11}$ así como la determinación de la precisión intermedia.

\section{MATERIAL Y MÉTODOS}

La verificación del desempeño se realizó por medio de un análisis comparativo para los inmunoanálisis quimioluminiscentes de micropartículas (CMIA) HIV Ag/ $\mathrm{Ab}$ Combo ${ }^{\mathrm{TM}}$, Anti-HCV ${ }^{\mathrm{TM}}$, y HBsAg Qualitative II $^{\mathrm{TM}}$ de la marca Architect Abbott ${ }^{\circledR}$, se utilizaron 40 muestras para cada ensayo y se procesaron ocho por día durante cinco días. Se tomaron como criterios de inclusión muestras con resultados en el rango dinámico (fuertemente positivas, débilmente positivas, débilmente negativas y fuertemente negativas). Se buscó la idealidad de utilizar 
Tabla 1: Esquema de una tabla de contingencia.

\begin{tabular}{|c|c|c|c|}
\hline \multirow[b]{2}{*}{$\begin{array}{l}\text { Método por } \\
\text { verificar }\end{array}$} & \multicolumn{2}{|c|}{$\begin{array}{c}\text { Método de comparación } \\
\text { (referencia) }\end{array}$} & \multirow[b]{2}{*}{ Total } \\
\hline & Reactivo & No reactivo & \\
\hline Reactivo & a & $b$ & $a+b$ \\
\hline No reactivo & c & $d$ & $c+d$ \\
\hline Total & $a+c$ & $b+d$ & $n$ \\
\hline \multicolumn{4}{|c|}{$\begin{array}{l}\text { Porcentaje concordancia total }=((a+d) / n)^{*} 100 \\
\text { Porcentaje concordancia positiva }=(a /(a+c))^{*} 100 \\
\text { Porcentaje concordancia negativa }=(d /(b+d))^{*} 100 \\
\text { Donde: } \\
\text { a = Resultados concordantes, reactivos entre el laboratorio SMNYL y el } \\
\text { laboratorio de referencia. } \\
b=\text { Resultados discordantes, reactivo en SMNYL y no reactivo en el } \\
\text { laboratorio de referencia. } \\
c=\text { Resultados discordantes, no reactivo en SMNYL y reactivo en el } \\
\text { laboratorio de referencia. } \\
d=\text { Resultados concordantes no reactivos entre el laboratorio SMNYL y } \\
\text { el laboratorio de referencia. } \\
n=\text { Número total de muestras evaluadas. }\end{array}$} \\
\hline
\end{tabular}

la mitad de muestras positivas y la mitad negativas; $\sin$ embargo, por el uso previsto del laboratorio y dado que nuestra población es mayormente sana, se estableció utilizar como mínimo 10 muestras reactivas. Para el ensayo Anti-HCV ${ }^{\mathrm{TM}}$ se utilizaron 13 muestras reactivas y 27 no reactivas, para el ensayo $\mathrm{HBsAg}$ Qualitative II $^{\mathrm{TM}}$ se utilizaron 12 muestras reactivas y 28 muestras no reactivas, mientras que para el ensayo HIV Ag/Ab Combo ${ }^{\mathrm{TM}}$ se utilizaron 10 muestras reactivas y 20 no reactivas. Los resultados obtenidos por el laboratorio SMNYL se compararon con los obtenidos por un tercero ya acreditado por la ema en dichos ensayos por medio de tablas de contingencia, a partir de las cuales se calcularon los porcentajes de concordancia total, concordancia positiva y concordancia negativa de acuerdo con las fórmulas que se muestran en la Tabla 1.

Se establecieron como criterios de aceptación concordancias $\geq 90 \%$ para cada parámetro si la comparación se realiza con la misma metodología y concordancias $\geq 80 \%$ si la comparación se realiza con metodología superior o prueba confirmatoria; en este último caso la concordancia total representa la eficiencia del método, la concordancia positiva representa la sensibilidad y la concordancia negativa representa la especificidad.

Adicional al análisis comparativo y dada la metodología de los ensayos se determinó la precisión intermedia con base en la guía ema-CENAM, ${ }^{9}$ se realizó repeticiones de dos materiales de control para cada ensayo durante 20 días (evaluación interserial). Para el análisis estadístico se determinó la media, la desviación estándar (DE) y el coeficiente de variación (CV).

Se establecieron dos criterios de aceptación para la determinación de precisión intermedia: 1) CV menor o igual al CV reportado por el fabricante para cada nivel de control en las mismas condiciones, 2) CV menor o igual al 0.33 del error total máximo permitido $(\mathrm{ETa})$ por coeficiente de variabilidad biológica porcentual (CVb), lo que corresponde a 0.33 (49\%) o $16.17 \%$ para los tres ensayos.

\section{RESULTADOS}

\section{Tablas de contingencia y concordancias}

A continuación se muestran las Tablas 2 a 4 de contingencia y los resultados de la determinación de concordancias para los tres ensayos inmunológicos cualitativos, en los cuales se obtuvieron concordancias de $100 \%$ para cada criterio, lo que nos permitió comprobar el desempeño documentado por el fabricante.

\section{Precisión}

Los resultados de las determinaciones de precisión intermedia se muestran en la Tabla 5. Para los tres ensayos se obtuvieron coeficientes de variación menores a los reportados por el fabricante en las mismas condiciones, así como menores a la fracción permitida del ETa basado en CVb, por lo que los tres ensayos fueron aceptados en ambos criterios.

\section{Tabla 2: Tabla de contingencia y concordancias} para el ensayo anti-HCV ${ }^{\mathrm{TM}}$.

\begin{tabular}{lccc} 
& \multicolumn{2}{c}{ Anti-HCV referencia } & \\
\cline { 2 - 3 } Anti-HCV SMNYL & Reactivo & No reactivo & Total \\
\hline Reactivo & 13 & 0 & 13 \\
No reactivo & 0 & 27 & 27 \\
Total & 13 & 27 & 40 \\
\hline & & & \\
Parámetros evaluados & $(\%)$ & $(\%)$ & Interpretación \\
\hline Concordancia total & 100 & 90 & $\begin{array}{c}\text { Aceptada } \\
\text { Concordancia positiva }\end{array}$ \\
Concordancia negativa & 100 & 90 & $\begin{array}{c}\text { Aceptada } \\
\text { Aceptada }\end{array}$ \\
\hline
\end{tabular}




\begin{tabular}{|c|c|c|c|}
\hline \multirow[b]{2}{*}{ HBSAg SMNYL } & \multicolumn{2}{|c|}{ HBSAg referencia } & \multirow[b]{2}{*}{ Total } \\
\hline & Reactivo & No reactivo & \\
\hline Reactivo & 12 & 0 & 12 \\
\hline No reactivo & 0 & 28 & 28 \\
\hline Total & 12 & 28 & 40 \\
\hline $\begin{array}{l}\text { Parámetros } \\
\text { evaluados }\end{array}$ & $\begin{array}{l}\text { Resultado } \\
(\%)\end{array}$ & $\begin{array}{c}\text { Criterio } \\
(\%)\end{array}$ & Interpretación \\
\hline Concordancia total & 100 & 90 & Aceptada \\
\hline $\begin{array}{l}\text { Concordancia } \\
\text { positiva }\end{array}$ & 100 & 90 & Aceptada \\
\hline $\begin{array}{l}\text { Concordancia } \\
\text { negativa }\end{array}$ & 100 & 90 & Aceptada \\
\hline
\end{tabular}

\section{Tabla 4: Tabla de contingencia y concordancias para el ensayo HIV Ag/Ab Combo ${ }^{\mathrm{TM}}$.}

\begin{tabular}{lccc} 
& \multicolumn{2}{c}{ HIV Ag/Ab Referencia } & \\
\cline { 2 - 3 } HIV Ag/Ab SMNYL & Reactivo & No Reactivo & Total \\
\hline Reactivo & 10 & 0 & 10 \\
No reactivo & 0 & 30 & 30 \\
Total & 10 & 30 & 40 \\
\hline Parámetros & Resultado & Criterio & Interpretación \\
evaluados & $(\%)$ & $(\%)$ & \\
\hline Concordancia total & 100 & 90 & Aceptada \\
Concordancia positiva & 100 & 90 & Aceptada \\
Concordancia negativa & 100 & 90 & Aceptada \\
\hline
\end{tabular}

\section{CONCLUSIONES}

El protocolo y los resultados que hemos presentado nos llevaron a lograr la acreditación de los tres ensayos, ya que se obtuvo evidencia objetiva para verificar el desempeño de acuerdo con el uso previsto. Los resultados fueron revisados en un ejercicio de auditoría por parte de la ema en 2018, en el que participaron un evaluador líder técnico y dos expertos técnicos. En dicho ejercicio revisaron, además de los resultados presentados en este artículo, las evidencias de respaldo, entre otras cosas, los resultados emitidos por los equipos, el estado de calibración y la trazabilidad de los calibradores, el control de calidad interno y el desempeño en los programas de evaluación externa de la calidad, lotes y fechas de caducidad del material empleado, así como los informes de resultados utilizados como referencia.

El interés por obtener el reconocimiento a la calidad en el laboratorio a través de la acreditación va en franco aumento en nuestro país; sin embargo, cuando iniciamos este largo y costoso proceso, el impacto económico que representa para la organización es sustancial y lograr el cumplimiento a los requisitos asociados a la verificación de los métodos de ensayo representa sin duda el gasto más significativo. Si bien es cierto que enfrentamos las dificultades de cumplir requisitos con un alto índice de complejidad, debemos buscar la forma más sencilla y adecuada para dar cumplimiento, sin perder de vista lo más importante y que se convierte en el verdadero

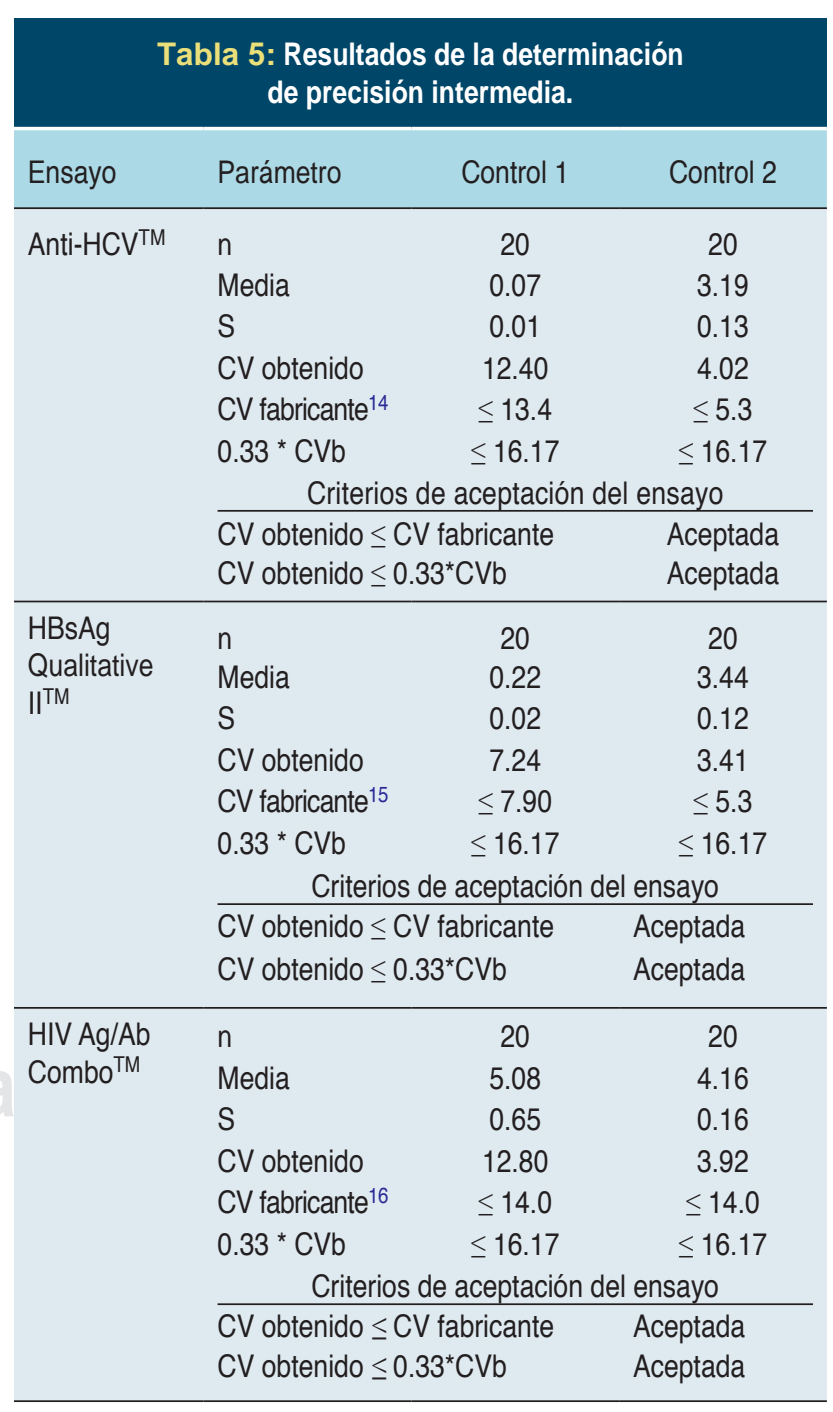


objetivo del laboratorio, proporcionar resultados oportunos y confiables.

Proponemos este protocolo como una alternativa, ya que a la par de ser sencillo y accesible, permite la obtención de evidencia objetiva para la verificación del desempeño.

\section{REFERENCIAS}

1. NMX-EC-15189-IMNC-2015 Laboratorios Clínicos-Requisitos de la calidad y competencia.

2. Entidad Mexicana de Acreditación AC. Disponible en: www.ema. org.mx.

3. Quintana S. The accreditation experience of clinical laboratories and blood banks in México. J Int Fed Clin Chem. 2015; 26: 259-263.

4. Laboratorio registrados en México con aviso de funcionamiento. Consultado en febrero 2019. Disponible en: https://datos.gob.mx/ busca/dataset/avisos-de-funcionamiento.

5. Catálogo de laboratorio acreditados en México. [Consultado en febrero 2019]. Disponible en: http://consultaema.mx:75/ Directorio_CL/Principal.aspx.

6. Garzón-Ā̄ba C. Quality management systems in the clinical laboratories in Latin America. J Int Fed Clin Chem. 2015; 26: 216-220.
7. Zamora-Palma A. Evaluación de tres directrices para la implementación de un sistema de gestión de la calidad. Rev Latinoam Patol Clin Med Lab. 2015; 62 (1): 11-15.

8. Carboni-Huerta R, Sáenz-Flor K. Acreditación ISO 15189 en América Latina: Percepción en laboratorios de la región. Rev Latinoam Patol Clin Med Lab. 2019; 66 (1): 143-153.

9. Delgado G. Validación y verificación de métodos de ensayo. Un dilema en los laboratorios de ensayos y en las auditorías de la acreditación. Universitas, 2009; 3 (2): 14-21.

10. Guía para la Validación y la Verificación de los Procedimientos de Examen Cuantitativos Empleados por el Laboratorio Clínico. EMACENAM, 2018.pp. 2-47.

11. User Protocol for Evaluation of Qualitative Test Performance; Approved Guideline. CLSI EP12-A2, 2008.

12. La Adecuación al Uso de los Métodos Analíticos-Una Guía de Laboratorio para Validación de Métodos y Temas Relacionados. EURACHEM, 2016

13. Garzón-Alba C. Validación, verificación o evaluación de métodos?... lo realmente importante es: ser una herramienta de seguridad para el paciente. Bogotá 2013.

14. Inserto Anti-HCV G5-9028/R11-2014. Disponible en: https://www. corelaboratory.abbott/tl/ous/technical-library.html.

15. Inserto HBsAg Qualitative II G4-5465/R05-2013. Available in: https://www.corelaboratory.abbott/t//ous/technical-library.html.

16. Inserto HIV Ag/Ab Combo G4-7762/R05-2014. Available in : https:// www.corelaboratory.abbott/tl/ous/technical-library.html. 